\title{
LA POBLACIÓN DE REGIONES RURALES EUROPEAS ANTE CUESTIONES SOBRE DESARROLLO INTERNACIONAL: UN ESTUDIO DE ENCUESTA
}

\author{
José María Marbán Prieto - Universidad de Valladolid \\ Noelia Santamaría-Cárdaba - Universidad de Valladolid \\ Luis Torrego Egido - Universidad de Valladolid
}

(D) $0000-0002-6561-6784$

(iD) $0000-0001-6864-9330$

(D) $0000-0002-2907-1158$

Recepción: 21.07.2021 | Aceptado: 16.10.2021

Correspondencia a través de ORCID: Noelia Santamaría-Cárdaba

iD 0000-0001-6864-9330

Citar: Marbán., JM, Santamaría-Cárdaba, N y Torrego, L (2021). La población de regiones rurales europeas ante cuestiones sobre desarrollo internacional: un estudio de encuesta. REIDOCREA, 10(32), 1-11. Financiación: European Commission - EuropeAid/134863/C/ACT/Multi

Área o categoría del conocimiento: multidisciplinar

\begin{abstract}
Resumen: El presente artículo pretende conocer la opinión de la población situada en regiones rurales europeas sobre diversas cuestiones relacionadas con el desarrollo internacional. La necesidad de disponer de una ciudadanía activa que abogue por un desarrollo humano, sostenible y justo hace que este estudio posea una relevancia especial. La metodología empleada fue cuantitativa, pues se realizó un estudio de encuesta en el que participaron 3026 personas de Bulgaria, Chipre, España, Grecia, Italia, Malta y Polonia. Los resultados indican que es necesario mejorar la formación de la población rural europea ante cuestiones globales y de desarrollo para promover una transformación social.
\end{abstract}

Palabra clave: Educación para la ciudadanía global

PEOPLE IN EUROPEAN RURAL REGIONS FACING INTERNATIONAL DEVELOPMENT ISSUES: A SURVEY STUDY

\begin{abstract}
This article seeks to ascertain the views of people living in Europe's rural regions on various issues related to international development. The need to have an active citizenship that advocates human, sustainable and fair development makes this study particularly relevant. The methodology used was quantitative, as a survey study was carried out in which 3026 people from Bulgaria, Cyprus, Greece, Italy, Malta, Poland and Spain participated. The results indicate that there is a need to improve the training of the European rural population on global and developmental issues in order to promote social transformation.
\end{abstract}

Keyword: Global Citizenship Education

\section{Introducción}

Esta investigación posee una relevancia especial al centrarse en tres aspectos indispensables para garantizar una transformación social: la población, el desarrollo sostenible y la Educación para la Ciudadanía Global; por ello, en este estudio se comprende que la población debe entender que posee el papel protagonista para impulsar un desarrollo económico, humano y ambiental más sostenible y justo. Autores como Cassen (1976) o Weeks (2020) señala que los debates académicos y de fuera de este ámbito están centrándose cada vez más en cuestiones ligadas a la población, siendo, por tanto, este estudio un aporte que enriquece los estudios existentes sobre ciudadanía y desarrollo. Concretamente, el presente estudio tiene como objetivo: conocer la opinión de la población rural europea ante cuestiones relacionadas con el desarrollo a nivel internacional.

A nivel internacional la ONU ha relegado a los anteriores Objetivos del Desarrollo del Milenio con los actuales Objetivos del Desarrollo Sostenible (ODS) fijados en la Agenda 2030 (ONU, 2015), lo cual hace que la consecución de los ODS sea un tema relevante actualmente en todos los ámbitos al tratar de dar respuesta a diversas problemáticas sociales (Sachs, 2012, 2020). Esta Agenda 2030 hace hincapié en la necesidad de que la población esté formada ante cuestiones ligadas con el desarrollo para impulsar un 
cambio hacia una sociedad más sostenible y justa (Schot, Boni, Ramirez, Steward, 2018; Mesa, 2019). De hecho, entre estos objetivos la educación es imprescindible para formar ciudadanos globales que aboguen por una transformación social está presente en el ODS 4.7 en el que se propone la población desarrolle "los conocimientos y aptitudes necesarios para promover el desarrollo sostenible, [...] los derechos humanos, la igualdad entre los géneros, la promoción de una cultura de paz y no violencia, la ciudadanía mundial y la apreciación de la diversidad cultural" (ONU, 2015, p.17).

En este sentido, la población debe estar adecuadamente formada para promover un cambio socioeconómico y ambiental a favor del desarrollo y de la igualdad entre países desarrollados y en desarrollo para hacer frente a la pobreza y la desigualdad (Wietzke, 2020). La Educación para la Ciudadanía Global (ECG) es, en este caso, una cuestión clave puesto que promueve la formación de una ciudadanía activa que piense críticamente, que comprenda las desigualdades sociales existentes tanto a nivel local como global e impulse una transformación hacia una sociedad más sostenible y justa (Bourn, 2011; Bourn, Hunt, Blum y Lawson, 2016; Pashby, Costa, Stein y Andreotti, 2020; Santamaría-Cárdaba, 2020).

Cabe recordar, como se ha señalado anteriormente, que este estudio se centra en descubrir la opinión de la población rural europea ante cuestiones ligadas al desarrollo y por ello, se deben resaltar varias cuestiones. Por un lado, el Consejo de Europa defiende la necesidad de formar una ciudadanía activa y comprende la ECG como "un proceso de crecimiento individual y colectivo que permite un cambio y una autotransformación" (Consejo de Europa, 2008: 19). De hecho, el Consejo Europeo en la Resolución del Consejo de 2001 sobre la ECG y la sensibilización de la opinión pública europea a favor de la cooperación para el desarrollo señala, en el sexto artículo, la importancia de proporcionar un mayor apoyo a la ECG y la política de comunicación sobre estas cuestiones para formar a la población ante diversas cuestiones sobre desarrollo (Unión Europea, 2001). Esto desvela que a nivel institucional en Europa se está dando importancia a que la población comprenda aspectos ligados al desarrollo y actúe favoreciendo un cambio socioeconómico respetuoso con el medio ambiente (Consejo de Europa, 2019).

Sin embargo, a pesar de que se han encontrado estudios que investigan sobre cuestiones como, por ejemplo: el crecimiento de la población europea y sus efectos para el medio ambiente (Weber \& Sciubba, 2019), el impacto demográfico de la inmigración para la población europea (Murphy, 2016) o aspectos ligados al desarrollo económico (Easterly \& Levine, 2016), hay una escasez de estudios en los que se ha buscado conocer la opinión de la población rural europea ante cuestiones sobre desarrollo (Abós, 2015). Por este motivo, el presente estudio es un aporte indispensable que permitirá descubrir si la población rural europea realmente actúa acorde con la perspectiva de la ECG y del desarrollo humano sostenible.

Por otro lado, otro motivo por el cual este estudio se centra en la población rural europea es que este estudio de encuesta emerge del proyecto Rural DEAR Agenda EYD-2015. En este proyecto europeo participaron ciudadanos que viven en localidades rurales de Bulgaria, Chipre, España, Grecia, Italia, Malta y Polonia. Este proyecto ha sido financiado por la Unión Europea y pretendía lograr dos cuestiones: 1) realizar un diagnóstico de la situación de la ECG en las zonas rurales de los siete países europeos participantes y 2), en base a estos resultados, realizar un Agenda Rural con actua ciones para que la población adquiera una competencia global adecuada que les permita hacer frente a las desigualdades sociales existentes y actuar a favor de un desarrollo equitativo (OCUVa, 2016). La Figura 1 es un mapa en el que se puede apreciar con detalle las regiones participantes de cada uno de los siete países que participaron en este estudio. 




Figure 1. Región rural de la población europea participante.

Por tanto, el proyecto europeo Rural DEAR Agenda EYD-2015 tiene como objetivo favorecer que la población europea que vive en zonas rurales sea solidaria, activa y esté sensibilizada comprendiendo que la responsabilidad para transformar la sociedad en una más sostenible, humana y justa es cosa de todos.

\section{Objetivos}

El presente estudio busca conocer la opinión de población rural de los siete países europeos ya nombrados ante diferentes cuestiones ligadas con el desarrollo y la ECG, por lo que se encuentra enmarcado en la fase de diagnóstico del Proyecto europeo citado anteriormente. A continuación, se pasa a detallar en el próximo apartado la metodología empleada para la realización de esta investigación.

\section{Método}

La metodología empleada en este estudio es cuantitativa, pues este enfoque se emplea en los estudios que del área de ciencias sociales (Gorard, 2003) y permiten realizar interpretaciones sobre los fenómenos sociales estudiados (Babones, 2016). En este caso, el diseño de la investigación fue no experimental ex post facto que pretendía realizar un estudio de encuesta con carácter predominantemente descriptivo. Por tanto, al tratarse de un estudio de encuesta se empleó como herramienta de recopilación de datos el cuestionario, el cual fue elaborado para este estudio y disponía de varios bloques de preguntas en los cuales algunas tenían formato de escala Likert y otros requerían de una respuesta múltiple.

La encuesta se tradujo a los idiomas de la población europea participante y se les facilitó la opción de completarles en papel o en formato digital para adaptarse a la situación de cada zona rural. El muestreo se realizó empleando un método mixto multietapa puesto que "facilita la elección de los elementos de la muestra aleatoria, especialmente cuando no se dispone de una lista de unidades de la población" (Grande y Abascal, 2005, p.78). Adicionalmente, se empleó un muestreo por cuotas en función de la edad, que abarcaban desde los 16 a los 74 años, el cual permitiera disponer de una muestra representativa de la población inscrita en los censos de las zonas rurales que se mostraron en la Figura 1 (Cardona, 2002). Cabe destacar que inicialmente se propuso que la muestra total fuera de 2800 cuestionarios cumplimentados, es decir, 400 por país; sin embargo, la muestra real superó estas expectativas alcanzando un total de 3026 participantes y permitió mantener el margen de error establecido para un nivel de confianza del $90 \%$ y $\mathrm{P}=\mathrm{Q}$ en el $\pm 5 \%$ para cada país y en $\pm 1.8 \%$ para el conjunto de toda la población. 


\section{Resultados}

\section{Datos sociodemográficos}

Los datos demográficos de las personas encuestadas fueron recopilados en función de las variables sexo y edad. La variable cualitativa sexo muestra que este estudio mantiene unos porcentajes equitativos al lograr la participación de mujeres (47\%) y hombres $(53 \%)$. Adicionalmente, la variable edad permite apreciar que se logró la participación en todas las cuotas de edades establecidas: 16-24 (796 respuestas), 2534 (570 respuestas), 35-44 (492 respuestas), 45-54 (460 respuestas), 55-64 (355 respuestas) y de 65-74 (352 respuestas). La distribución de la población europea participante en función de su edad y su sexo se puede apreciar en la Figura 2.

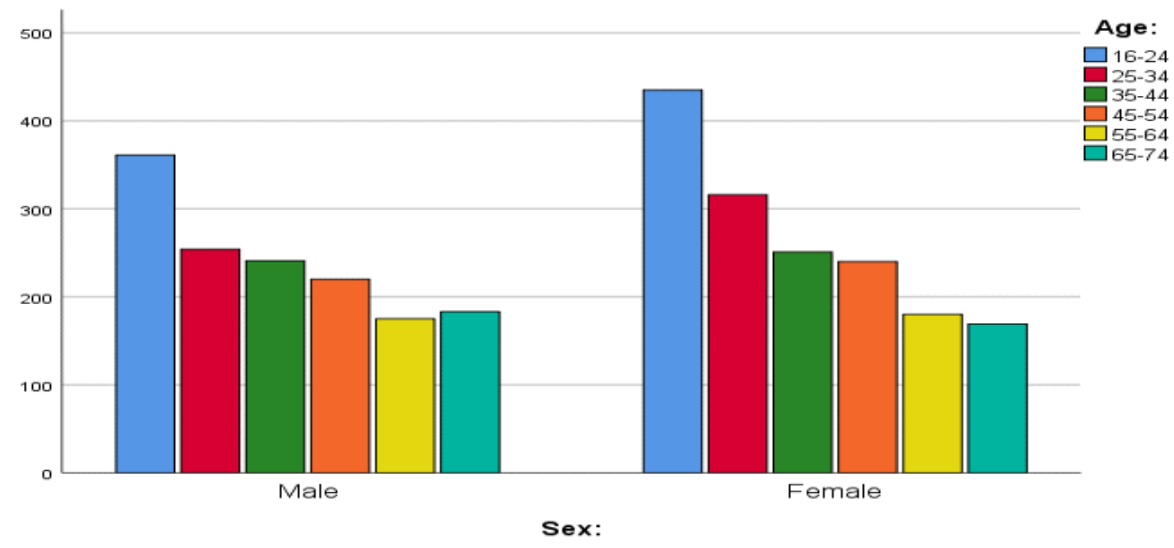

Figura 2. Distribución de los participantes en función del sexo y la edad.

\section{Problemas del mundo y causas de la pobreza}

La primera pregunta pretendía conocer cuáles son los problemas más importantes del mundo y sus causas según la percepción de la población rural europea. Por un lado, se pueden apreciar en la Figura 3 los problemas que la población considera más relevantes a nivel mundial. Como se puede apreciar, las personas participantes señalan como problemáticas más relevantes los conflictos armados (42,9\%), la crisis económica y el desempleo $(39,3 \%)$ y el terrorismo $(30,2 \%)$. Sin embargo, resulta llamativo que el acceso a saneamientos $(3,9 \%)$ o al agua potable $(5,2 \%)$ y el individualismo que conlleva una pérdida de valores $(7,7 \%)$, el cual está cada vez más presente en la sociedad actual, presentan frecuencias muy inferiores.

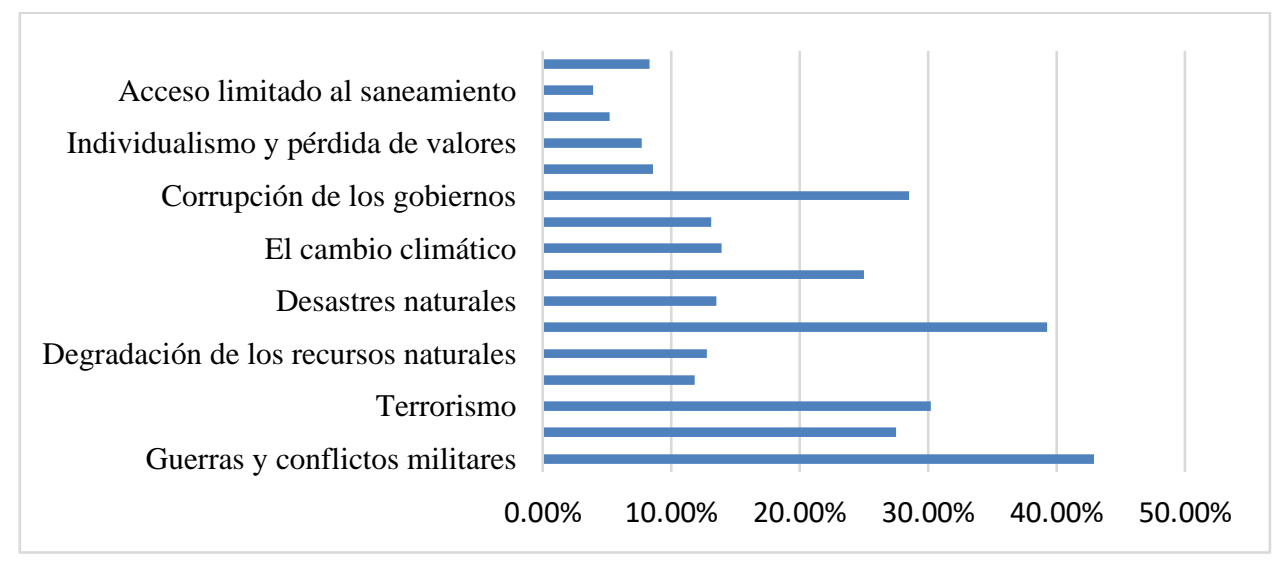

Figura 3. Frecuencias relativas a los problemas existentes en el mundo. 
Respecto a las respuestas proporcionadas sobre los problemas más relevantes, se debe señalar que se encuentran diferencias significativas en función del sexo o la edad en varias cuestiones: 1) los conflictos bélicos presentan diferencias significativas en función de la edad (Sig. 0,047), siendo los jóvenes que tienen entre 16 y 24 años los que señalan con mayor frecuencia esta alternativa; 2) el terrorismo es un problema más valorado por los hombres que por las mujeres (Sig. 0,001); 3) las desigualdades entre hombres y mujeres presentan diferencias significativas en función del sexo (Sig. 0,000) siendo las mujeres las que señalan esta problemática en mayor medida $(66,9 \%)$; 4) los desastres naturales presentan diferencias en función de la edad (Sig. 0,012) siendo la población de menor edad la que más selecciona esta problemática; 5) el cambio climático es considerado un problema en mayor medida por los hombres $(52,1 \%)$ por lo que hay diferencias en función del sexo (Sig. 0,036); la falta de alimentos es una problemática que tienden a marcar más las mujeres $(58,8 \%)$ que los hombres $($ Sig. 0,035$)$ y es especialmente valorado en la población que posee entre 16 y 34 años por lo que también hay diferencias en función de la edad (Sig. 0,017).

Por otro lado, la Figura 4 muestra la distribución de frecuencias de las respuestas obtenidas sobre las causas de los problemas internacionales y la pobreza. En este caso, las causas marcadas con mayor frecuencia por la población europea participante son: las relacionadas con las guerras y el desequilibrio político (40\%), la corrupción (40\%) y el paro (29\%). Mientras que las que adquirieren valoraciones inferiores son las condiciones de trabajo (6\%), las políticas de las empresas ricas $(6,7 \%)$ y la vulnerabilidad ante los desastres naturales $(9,6 \%)$.

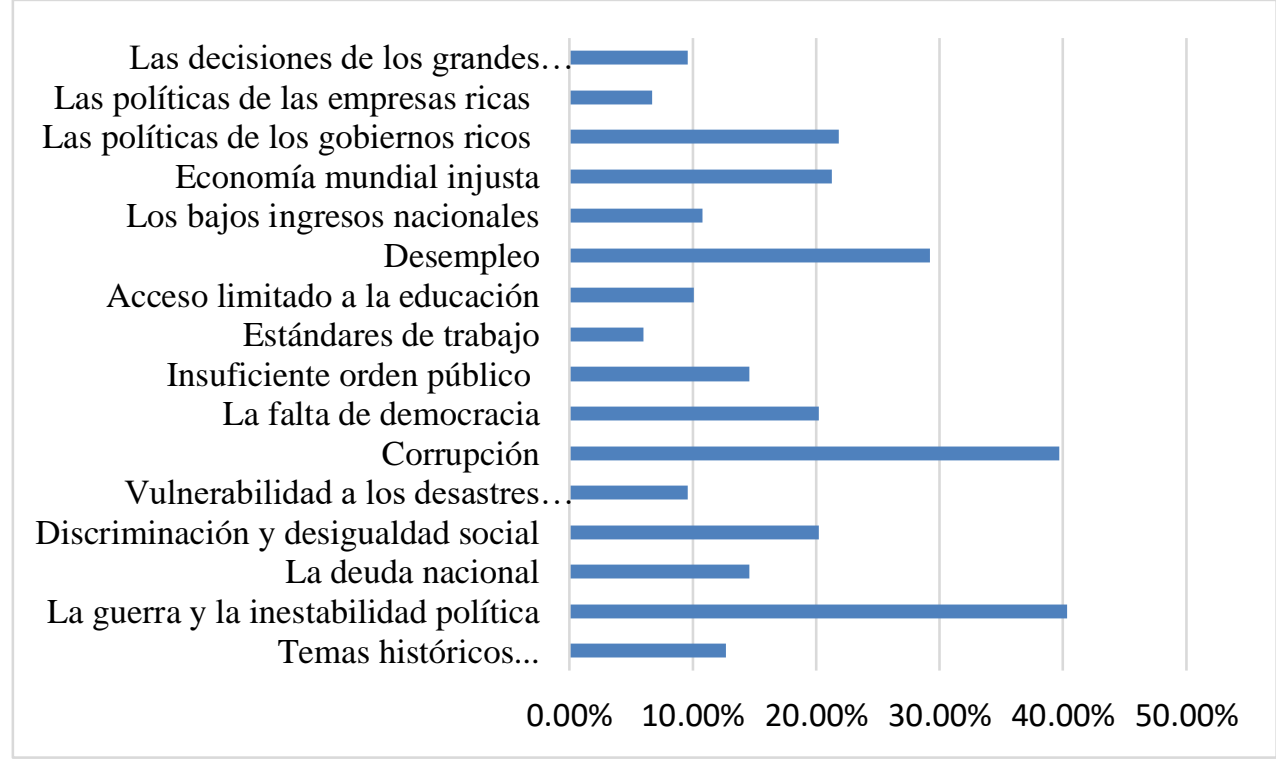

Figura 4. Frecuencias relativas a las causas de los problemas internacionales.

En relación con las causas que generan los problemas sociales existentes, se pasan a resaltar las diferencias significativas encontradas en función del sexo y/o la edad: 1) los asuntos históricos presentan diferencias significativas en función del sexo (Sig. 0,010) y en función de la edad $(0,000) ; 2$ ) la deuda nacional muestra diferencias en función de la edad de la población (Sig. 0,007); 3) la falta de democracia presenta diferencias significativas en función del sexo de los participantes (Sig. 0,046); 4) el desempleo es un problema que presenta diferencias significativas en función de la edad (Sig. 0,000) siendo la población que posee entre 16 y 24 años la que lo señala en mayor medida (32\%); 5) los bajos ingresos nacionales presentan diferencias en función del sexo (Sig. 0,007 ) siendo las mujeres las que marcan esta alternativa en mayor medida que los hombres; 6) la injusta economía mundial es una cuestión que muestra diferencias dependiendo de la edad de la población (Sig. 0,000) y esto mismo ocurre 7) con las 
políticas de las empresas ricas (Sig. 0,008) y 8) con las decisiones de los grandes lobbies (Sig. 0,000).

\section{Conocimientos sobre diversos temas de ECG}

Esta pregunta de la encuesta buscaba conocer los conocimientos que se posee la población sobre diversos los temas de ECG y que eran medidos en una escala de 1 a 5 . En este caso, se deben señalar especialmente las preguntas relacionadas con los objetivos del desarrollo ( $\overline{\mathrm{X}}=2.19)$, los proyectos y objetivos de la cooperación para el desarrollo $(\overline{\mathrm{X}}=2.42)$ y los vínculos entre desarrollo y educación $(\overline{\mathrm{X}}=2.9)$ dado que adquieren valores llamativamente bajos.

Adicionalmente, como se aprecia en la Cuadro 1 todos los temas de ECG propuestos presentan diferencias significativas en función de la edad de la población; mientras que las diferencias significativas en base al sexo se aprecian en las siguientes opciones: las estructuras y preocupaciones del comercio justo, los objetivos de las organizaciones antiglobalización, los orígenes de la deuda externa, los programas de apadrinamiento de niños y las relaciones entre educación y desarrollo.

Cuadro 1. Comparativa aplicando ANOVA sobre los temas de ECG.

\begin{tabular}{lcc}
\hline Comparación aplicando ANOVA & Sig. Sexo & Sig. Edad \\
\hline Los objetivos y proyectos de cooperación para el desarrollo &, 604 &, 000 \\
\hline Los Objetivos de Desarrollo del Milenio &, 085 &, 000 \\
\hline Las estructuras y preocupaciones del comercio justo &, 010 &, 000 \\
\hline Las normas de la ayuda humanitaria &, 585 &, 000 \\
\hline Los objetivos de las organizaciones antiglobalización &, 009 &, 000 \\
\hline Los orígenes de las deudas externas &, 000 &, 000 \\
\hline La política de compras éticas &, 293 &, 000 \\
\hline Los desafíos para el desarrollo humano sostenible &, 260 &, 000 \\
\hline Los programas de apadrinamiento de niños &, 001 &, 000 \\
\hline Los vínculos entre la educación y el desarrollo &, 010 &, 000
\end{tabular}

\section{Relevancia de las áreas de cooperación internacional}

La presente cuestión pretendía conocer a qué aspectos de la cooperación internacional para el desarrollo otorga mayor relevancia para la población rural europea encuestada. Como se puede apreciar en la Figura 5 el área considerado más importante es la erradicación de la pobreza (55\%); mientras que aspectos como la cohesión social y el desempleo, la protección de los derechos humanos y del medio ambiente o la atención de las necesidades básicas de todas las personas adquieren una menor relevancia para las personas participantes. Adicionalmente, teniendo presente la situación de Europa en el momento de realización de este estudio, resulta llamativo que los aspectos relacionados con la inmigración y el asilo político solamente son considerados esenciales por un $9 \%$ de los participantes.



Figura 5. Frecuencia de la importancia de las áreas de cooperación internacional. 
Antes de pasar a mostrar los resultados de la siguiente cuestión relativa a los problemas de los países empobrecidos, se deben señalar que se han apreciado diferencias en las siguientes alternativas: 1) el desarrollo de infraestructuras presenta diferencias en función del sexo de los participantes (Sig. 0,030) siendo los hombres los que dan mayor relevancia a esta cuestión $(52,2 \%) ; 2$ ) la protección medioambiental muestra diferencias en función de la edad de la población (Sig. 0,019); 3) las tecnologías de energía sostenible presenta diferencias significativas dependiendo de la edad (Sig. 0,007) y lo mismo ocurre con 4) el apoyo para las reformas institucionales (Sig. 0,010); 5) la cohesión social y el desempleo muestra diferencias significativas tanto en función del sexo de los participantes (Sig. 0,000) como de su edad (Sig. 0,007); 6) la erradicación de la pobreza también tiene diferencias en función de la edad (Sig. 0,028) y del sexo de la población encuestada (Sig. 0,000).

\section{Propuestas para reducir los problemas de los países empobrecidos}

La última cuestión pretendía conocer qué propuestas de las cinco establecidas eran más valoradas por la población europea para reducir los problemas de los países empobrecidos. En esta cuestión, todas las soluciones planteadas son marcadas por las personas participantes con escaso margen de duda, pues la media de todas ellas se encuentra por encima de 3,6. Sin embargo, la última opción genera más dudas estableciendo su media en 2,9. Esto indica que la población considera que es necesario que se produzcan transformaciones sociopolíticas y culturales a nivel internacional para ayudar tanto de manera personal como desde los gobiernos a solventar las desigualdades existentes en los países más empobrecidos.

Respecto a las diferencias en las respuestas obtenidas en base al sexo o la edad, como se puede apreciar en la Cuadro 2 la población participante presenta diferencias significativas prácticamente en todas las cuestiones planteadas.

Cuadro 2. Comparativa aplicando ANOVA sobre los problemas de los países empobrecidos.

\begin{tabular}{lcc}
\hline Comparación aplicando ANOVA & Sig. Sexo & Sig. Edad \\
\hline La ayuda internacional para ellos debería incrementarse &, 001 &, 000 \\
\hline El actual sistema económico mundial debe ser modificado &, 000 &, 007 \\
\hline Nuestros hábitos como consumidores deben cambiar &, 000 &, 000 \\
\hline $\begin{array}{l}\text { Los cambios políticos, sociales y económicos deben ser promovidos } \\
\text { dentro de sus fronteras }\end{array}$ &, 002 &, 011 \\
\hline Nadie del extranjero debe intervenir &, 402 &, 000 \\
\hline
\end{tabular}

Tras realizar el análisis de datos, se pasa a desarrollar en el siguiente epígrafe la discusión de estos con la literatura académica.

\section{Discusión}

La población participante destaca a los conflictos armados como el principal problema mundial que atañe a toda la sociedad. A juicio de autores como Fröhlich (2018), Fröhlich y Jungblut (2018) o Torres-Soriano (2011), los medios de comunicación tienen una gran influencia en la población y los conflictos armados son una de las temáticas a las que se suele dar cobertura para informar a la población. De hecho, este tratamiento mediático del conflicto hace que la ciudadanía sea más consciente de la relevancia de esta problemática que de otras a las que no se les presta tanta atención y, por tanto, tienden a presentar una menor influencia en la opinión de la población (Califano, 2015).

El desempleo y la crisis económica son otros de los conflictos más relevantes para la población encuestada, los cuales están relacionados con el problema existente en los países de la Unión Europea de la economía sumergida dado que siempre ha habido "una preocupación por su existencia, falta de control, tamaño y evolución, y no cabe 
duda de los numerosos efectos negativos que tiene en la economía, [...] y la incertidumbre que genera en los ciudadanos y en las empresas" (García, 2015, p.38). Por su parte, Torrego (2002) realizó a estudiantes universitarios esta misma pregunta obteniendo unos resultados similares, ya que entre los problemas principales el alumnado encuestado señalaba el terrorismo (42,3\%), la corrupción $(34,1 \%)$ y el paro $(21,5 \%)$. Sin embargo, el estudiantado universitario marca como principal problema mundial al deterioro medioambiental $(56,2 \%)$.

Adicionalmente, las principales causas de la pobreza según la población rural europea encuestada son las guerras, el desequilibrio político y la corrupción. La estrecha relación entre corrupción y pobreza es una cuestión ya destacada en múltiples investigaciones (Fisman y Miguel, 2010; Gupta, Davoodi y Alonso-Terme, 2002; Gundlach y Paldam, 2009), las cuales se han realizado en diversos países como, por ejemplo: Reino Unido (Gutterman, 2017), África (Ajisafe, 2016; Evans y Kelikume, 2019; Salahuddin, Vink, Ralph, y Gow, 2020) o China (Wu y Christensen, 2020). Esto revela que la corrupción política es una inquietud latente en la población a nivel internacional, pues genera pobreza y desequilibrios en los territorios en los que tiene lugar.

Respecto a los conocimientos sobre temáticas relacionadas con la ECG, este estudio reveló que a pesar de la relevancia innegable de los ODS (Sachs, 2015; Sachs, SchmidtTraub, Mazzucato, Messner, Nakicenovic y Rockström, 2019) no son especialmente conocidos por la población. Este bajo conocimiento de los ODS está latente en estudios como el realizado por Zamora-Polo, Sánchez-Martín, Corrales-Serrano y EspejoAntúnez (2019) a estudiantes universitarios. Por este motivo, autores como Annan-Diab y Molinari, (2017), Giangrande, White, East, Jackson, Clarke, Saloff, y Penha (2019) o Rieckmann (2017) señalan la importancia de impulsar una educación para el desarrollo sostenible que haga hincapié en los ODS.

En relación con las áreas de cooperación internacional más relevantes, la población rural europea señala como la más importante la erradicación de la pobreza. En este sentido, Tassara (2013) estudia el diálogo euro-latinoamericano existente en relación con la cooperación internacional y argumenta que "los gobiernos confirman su compromiso con la reducción de las desigualdades y de la pobreza" (Tassara, 2013, p.23), lo cual puede incidir en que la población de más relevancia a esta cuestión. No obstante, se debe resaltar que el discurso dominante sobre la lucha contra la pobreza no está del todo claro, ya que "contribuye a la creación de escasez y oculta el funcionamiento de esos mecanismos, desempoderando así a los pobres y engañando a las personas de buena voluntad" (Yapa, 1996, p.707).

En última instancia, los problemas de los países empobrecidos según la población europea deben solventarse a través de cambios sociales, culturales y políticos. De hecho, autores como Birdsall, Rodrik y Subramanian (2005) o Mkandawire, Kayembe, y Katombosola (2016) señalan que es importante tener en cuenta los contextos políticos, históricos y económicos para actuar ayudando a los países empobrecidos e impulsar la implementación de la agenda para el desarrollo para tratar de frenar las desigualdades existentes. No obstante, este tipo de cambios no deben recudirse a los gobiernos, sino que es necesario que la población esté formada como ciudadanos globales y participen activamente actuando frente a las injusticias y las desigualdades sociales existentes a nivel global (Birt, Poland, Csipke y Charlesworth, 2017; Kenny y Taylor, 2016; País y Costa, 2020). Por tanto, tal y como concluían Santamaría-Cárdaba, Marbán y Torrego (2019, p.195) en su estudio "la población está sensibilizada, pero no se implica de forma profunda en cuestiones relativas al desarrollo, lo que permite apreciar que sigue siendo necesario seguir avanzando en el diseño de estrategias efectivas de ECG en estas zonas rurales europeas". 


\section{Conclusiones}

Los resultados de este estudio revelan que la opinión de la población rural europea participante con relación a diversas cuestiones ligadas al desarrollo internacional. Por un lado, la población europea encuestada considera que los principales problemas internacionales son los conflictos armados y la crisis económica. Y a su vez, consideran que la pobreza generalmente está causada por los conflictos bélicos, la corrupción y los desequilibrios políticos. De hecho, la propuesta para reducir los problemas de los países empobrecidos más valorada por los participantes es la necesidad de que se produzcan cambios sociales, políticos y culturales para ayudar a frenar las desigualdades. Por tanto, la población sitúa a los gobiernos como el eje central que debe actuar correctamente de forma justa y promoviendo la transformación social que abogue por el cumplimiento de los ODS.

Adicionalmente, los resultados permiten concluir que la ciudadanía rural europea debe mejorar su formación en cuestiones ligadas a la ECG, pues los bajos conocimientos sobre cuestiones tan relevantes a nivel internacional como los ODS, los proyectos sobre cooperación al desarrollo o la relación entre desarrollo y educación deben de ser solventados. Además, las diferencias encontradas en función de la edad de los participantes revelan la necesidad de adecuar las propuestas formativas para llegar adecuadamente a toda la población.

Por otro lado, respecto a las áreas de cooperación internacional, la ciudadanía participante considera que la erradicación de la pobreza es la más relevante, pero esto quizá pueda deberse a que es una cuestión que está presente en el diálogo social y mediático. En este sentido, resulta llamativo que otros aspectos como la protección de los derechos humanos o del medio ambiente tengan una menor relevancia siendo cuestiones de una relevancia innegable para toda la población.

Por consiguiente, la población rural europea muestra la importancia de que se les proporcione una correcta formación por lo que se deberían implementar propuestas formativas de ECG adecuadas a la edad de las personas a las que van dirigidas. Asimismo, se puede concluir que tanto los gobiernos, como la ciudadanía tiene un papel innegable para favorecer el desarrollo internacional y hacer frente a las desigualdades sociales existentes impulsando una transformación hacia una sociedad defienda los derechos humanos y actúe tratando de frenar la pobreza y las injusticias.

\section{Referencias}

Abós, P. (2015). El Modelo de Escuela Rural ¿Es un Modelo Transferible a Otro Tipo de Escuela?. Educação \& Realidade, 40(3), 667-684.

Ajisafe, R. A. (2016). Corruption and poverty in Nigeria: evidence from ardl bound test and error correction model. Journal of Emerging Trends in Economics and Management Sciences, 7(3), 156-163.

Annan-Diab, F., \& Molinari, C. (2017). Interdisciplinarity: Practical approach to advancing education for sustainability and for the Sustainable Development Goals. The International Journal of Management Education, 15(2), 73-83.

Babones, S. (2016). Interpretive quantitative methods for the social sciences. Sociology, 50(3), 453-469.

Birdsall, N., Rodrik, D., \& Subramanian, A. (2005). How to help poor countries. Foreign affairs, 136-152.
Birt, L., Poland, F., Csipke, E., \& Charlesworth, G. (2017). Shifting dementia discourses from deficit to active citizenship. Sociology of Health \& IIness, 39(2), 199-211.

Bourn, D. (2011). Discourses and Practices around Development Education: From Learning about Development to Critical Global Pedagogy. Policy \& Practice: A Development Education Review, 13, 11-29.

Bourn, D., Hunt, F., Blum, N., \& Lawson, H. (2016). Primary Education for Global Learning and Sustainability. York: Cambridge Primary Review.

Califano, B. (2015). Los medios de comunicación, las noticias y su influencia sobre el sistema político. Revista mexicana de opinión pública, (19), 61-78.

Cardona, C. (2002). Introducción a los Métodos de Investigación en Educación. Madrid: EOS.

Cassen, R. H. (1976). Population and development: a survey. World Development, 4(10-11), 785-830. 
Council of Europe. (2008). Global Education Guidelines: A Handbook for Educators to Understand and Implement Global Education. Lisbon: North-South Centre of the Council of Europe.

Council of Europe. (2019). Global Education Guidelines: Concepts and Methodologies on Global Education for Educators and Policy Makers. Lisbon: North-South Centre of the Council of Europe.

Easterly, W., \& Levine, R. (2016). The European origins of economic development. Journal of Economic Growth, 21(3), 225-257.

Evans, O., \& Kelikume, I. (2019). The impact of poverty, unemployment, inequality, corruption and poor governance on Niger Delta militancy, Boko Haram terrorism and Fulani Herdsmen attacks in Nigeria. International Journal of Management, Economics and Social Sciences, 8(2), 58-80.

Fisman, R., \& Miguel, E. (2010). Economic gangsters: Corruption, violence, and the poverty of nations. Nueva Jersey: Princeton University Press.

Fröhlich, R. (Ed.). (2018). Media in War and Armed Conflict: Dynamics of Conflict News Production and Dissemination. London: Routledge.

Fröhlich, R., \& Jungblut, M. (2018). Between factoids and facts: The application of 'evidence' in NGO strategic communication on war and armed conflict. Media, War \& Conflict, 11(1), 85-106.

García, J. (2015). Economía sumergida y relaciones laborales en Europa. Revista latinoamericana de derecho social, 21, 31-59.

Giangrande, N., White, R. M., East, M., Jackson, R., Clarke, T., Saloff, M., \& Penha, G. (2019). A competency framework to assess and activate education for sustainable development: Addressing the UN sustainable development goals 4.7 challenge. Sustainability, 11(10), 2832

Grande, I., y Abascal, E. (2005). Análisis de encuestas. Madrid: ESIC.

Gorard, S. (2003). Quantitative methods in social science research. Londres: A\&C Black.

Gundlach, E., \& Paldam, M. (2009). The transition of corruption: From poverty to honesty. Economics Letters, 103(3), 146-148.

Gupta, S., Davoodi, H., \& Alonso-Terme, R. (2002). Does corruption affect income inequality and poverty? Economics of governance, $3(1), 23-45$

Gutterman, E. (2017). Poverty, corruption, trade, or terrorism? Strategic framing in the politics of UK anti-bribery compliance. The British Journal of Politics and International Relations, 19(1), 152171.

Kenny, S., \& Taylor, M. (2016). Challenging the third sector: Global prospects for active citizenship. Bristol: Policy Press.

Mesa, M. (2019). La Educación para la Ciudadanía Global y los Objetivos de Desarrollo Sostenible: Una Agenda para la Transformación Social. Revista Internacional De Educación Para La Justicia Social, 8(1), 7-11.

Mkandawire, M. T., Kayembe, A. E., \& Katombosola, B. (2016). Does development aid help poor countries to develop? a critical analysis of Africa's situation. American Scientific Research Journal for Engineering, Technology, and Sciences, 26(2), 53-66.

Murphy, M. (2016). The impact of migration on long-term European population trends, 1850 to present. Population and Development Review, 225-244.

Observatorio de Cooperación Internacional para el Desarrollo de la Universidad de Valladolid (OCUVa). (2016). Resumen ejecutivo del diagnóstico de la Educación para el Desarrollo en el ámbito rural europeo: resumen ejecutivo. Valladolid: OCUVa.

Pais, A., \& Costa, M. (2020). An ideology critique of global citizenship education. Critical Studies in Education, 61(1), 1-16.

Pashby, K., da Costa, M., Stein, S., \& Andreotti, V. (2020). A metareview of typologies of global citizenship education. Comparative Education, 56(2), 144-164.

Rieckmann, M. (2017). Education for sustainable development goals: Learning objectives. París: UNESCO.

Sachs, J. (2012). From millennium development goals to sustainable development goals. The Lancet, 379(9832), 2206-2211.

Sachs, J. (2015). Achieving the sustainable development goals. Journal of International Business Ethics, 8(2), 53.

Sachs, J., Schmidt-Traub, G., Mazzucato, M., Messner, D., Nakicenovic, N., \& Rockström, J. (2019). Six transformations to achieve the sustainable development goals. Nature Sustainability, 2(9), 805-814.

Sachs, J. (2020). The Ages of Globalization: Geography, Technology, and Institutions. Nueva York: Columbia University Press.

Salahuddin, M., Vink, N., Ralph, N., \& Gow, J. (2020). Globalisation, poverty and corruption: Retarding progress in South Africa. Development Southern Africa, 37(4), 617-643.

Santamaría-Cárdaba, N. (2020). Looking for the way out of the labyrinth: analysis of the definition of development education. Educação e Pesquisa, 46, 1-17.

Santamaría-Cárdaba, N., Marbán, J., \& Torrego, L. (2019). Diagnóstico de la Educación para el Desarrollo en áreas rurales: un análisis correlacional de las actitudes de la población europea. Revista de fomento social, (294), 177-200.

Schot, J., Boni, A., Ramirez, M., \& Steward, F. (2018). Addressing the sustainable development goals through transformative innovation policy.

Tassara, C. (2013). Cooperación para el desarrollo, relaciones internacionales y políticas públicas. Teorías y prácticas del diálogo euro-latinoamericano. Medellín: Ediciones UNAULA.

Torres-Soriano, M. R. (2011). Guerras Youtube: el impacto de las nuevas tecnologías de la información en el tratamiento mediático de los conflictos armados. Cuadernos de estrategia, (148), 129-157.

Weber, H., \& Sciubba, J. D. (2019). The effect of population growth on the environment: Evidence from European regions. European Journal of Population, 35(2), 379-402. 
Wietzke, F. B. (2020). Poverty, Inequality, and Fertility: The Contribution of Demographic Change to Global Poverty Reduction. Population and Development Review, 46(1), 65-99.

Wu, S., \& Christensen, T. (2020). Corruption and Accountability in China's Rural Poverty Governance: Main Features from Village and Township Cadres. International Journal of Public Administration, 111.

ONU. (2015). Transforming our World: The 2030 Agenda for Sustainable development. Nueva York: United Nations.

Unión Europea. (2001). Sesión $\mathrm{n}^{0} 2383$ del Consejo DESARROLLO -. Bruselas, 8 de noviembre de 2001.

Viña, J. G. (2015). Economía sumergida y relaciones laborales en Europa. Revista latinoamericana de derecho social, 21, 31-59.

Weeks, J. R. (2020). Population: An introduction to concepts and issues. Boston: Cengage Learning.

Yapa, L. (1996). What causes poverty?: A postmodern view. Annals of the Association of American Geographers, 86(4), 707-728.

Zamora-Polo, F., Sánchez-Martín, J., Corrales-Serrano, M., \& Espejo-Antúnez, L. (2019). What do university students know about sustainable development goals? A realistic approach to the reception of this UN program amongst the youth population. Sustainability, 11(13), 3533. 